\title{
INFLAMMATORY PRIMING OF THE FETAL SHEEP METABOLISM PREDICTS BRAIN DAMAGE
}

M. Keller ${ }^{1}$, D. Enot ${ }^{1}$, J. Dean ${ }^{2}$, H. Bolouri ${ }^{2}$, Y. van de Looi ${ }^{3}$, G. Lodygensky ${ }^{3}$, I. Kjellmer ${ }^{4}$, H. Hagberg ${ }^{5}$, S. Sizonenko ${ }^{3}$, C. Mallard ${ }^{2}$

${ }^{I}$ Department of Pediatrics, University Hospital Essen, Essen, Germany, ${ }^{2}$ Perinatal Centre, Gothenburg, Sweden, ${ }^{3}$ Department of Pediatrics, University Geneva, Geneva, Switzerland, ${ }^{4}$ Department of Pediatrics, ${ }^{5}$ Departments of Obstetrics and Gynecology, Göteborg University, Gothenborg, Sweden

Background and aims: Prenatal inflammation is currently being seen as one important factor of brain injury in preterm infants. Currently there is a lack of an early biomarker of brain injury. In addition it is not known how prenatal inflammation influences fetal metabolism in short and long-term.

\section{Here, we}

1) characterise the fetal metabolic response to intrauterine inflammation

2) identify metabolic markers of brain injury.

Methods: Fetuses were randomized to i.v. bolus infusion of either saline-vehicle $(n=7)$ or 200ng E.coli LPS $(\mathrm{n}=9)$ at $102.5 \pm 0.5$ days of gestation. Blood samples were collected at baseline $2 \mathrm{~h}, 6 \mathrm{~h}$ hours and daily up to 10 days for metabolite quantification. Metabolites were correlated to findings in MRI, EEG and histopathology.

Results: LPS induced a two-phase pattern in metabolite changes. Within the first 3 days, 121 metabolites were impacted (immune response, energy metabolism and tissue injury). Thereafter, a transient period (46days) without changes compared to baseline, was followed by a second phase marked by an opposite regulation of energy metabolites and inflammatory markers. At the multivariate level, the two-phase characteristics of the metabolite response to LPS was strongly associated with white/grey matter volumes and the number of oligo-2 positive cells at $2 \mathrm{~h}$-to 2 days and at 6-9days.

Conclusions: In this first in-utero analysis we detected a significant impact of LPS on fetal metabolism in both the short and long-term. Thus we hypothesize that there is specific inflammatory priming of the fetal metabolism in the blood, as supported by the bi-phasic pattern of metabolites, which correlate with brain injury. 